\title{
Les gènes du vieillissement
}

Cela fait bien longtemps que nous proposons, dans $\mathrm{m} / \mathrm{s}$, une hypothèse du vieillissement qui en fait la contrepartie de la transformation maligne. Selon ce schéma, le cancer serait la conséquence d'un déséquilibre, aux dépens des "antioncogènes ", de l'équilibre normal oncogènes/anti-oncogènes, alors que la sénescence pourrait être la conséquence du phénomène inverse $(\mathrm{m} / \mathrm{s}$ $n^{\circ} 2$, vol. 1 , p. $205 ; n^{\circ} 7$, vol. 2, p. $404 ; n^{\circ} 8$, vol. 2, p. $467 ; n^{\circ} 3$, vol. $6, p$. ?).

L'une des expériences fondamentales qui a débouché sur la notion d'antioncogène est issue des techniques de génétique somatique : le phénotype non transformé est dominant sur le phénotype cancéreux. Cela signifie que l'hybridation somatique entre des cellules cancéreuses et des cellules normales aboutit à des hybrides non transformés, à moins que ne soient perdus certains chromosomes issus des cellules parentales normales. Or la même chose est vraie concernant la sénescence, qui est le plus souvent dominante sur le phénotype "jeune" ou "immortalisé ". Cette notion, connue depuis plusieurs années, vient d'être à nouveau illustrée par un article issu d'une équipe du NIH (Triangle Park, NC, USA) [1]. Des cellules immortelles de hamster ont été hybridées avec des fibroblastes humains sénescents. La plupart des clones hybrides présentaient un phénotype sénescent, sauf ceux ayant perdu le chromosome 1 humain. Il est possible d'introduire dans des cellules un seul chromosome provenant d'une autre population cellulaire, en utilisant la technique des microcellules (microcells) : un traitement particulier de cellules en métaphase provoque leur éclatement en un grand nombre de petites vésicules cellulaires ne contenant qu'une partie des chromosomes, voire un seul. Ces microcellules sont alors fusionnées avec des cellules normales comme $\mathrm{m} / \mathrm{s} n^{\circ} 4$ vol. 6 , avril 90 dans des expériences habituelles d'hybridation cellulaire. Dans ces conditions, les auteurs ont démontré que le chromosome 1, à lui tout seul, suffisait à établir un phénotype sénescent [1]. Cette expérience ne signifie pas que le gène du vieillissement est localisé sur le chromosome 1. En effet, des expériences plus anciennes, consistant à hybrider entre elles des cellules immortalisées provenant de lignées différentes et à analyser les clones hybrides sénescents observés, ont permis de suggérer l'existence d'au moins quatre groupes de complémentation, c'est-à-dire de gènes qui devraient être co-exprimés pour induire la sénescence chez l'homme ; le chromosome 1 correspondrait à l'un de ces groupes.

La base génétique de la sénescence vient d'être illustrée de façon toute différente par Walter J. Gehring et son équipe, du Biozentrum de Bâle (Suisse) [2]. W.J. Gehring travaille depuis de nombreuses années sur le développement de la drosophile et il est d'ailleurs un très sérieux espoir de prix Nobel pour ses découvertes dans le domaine des gènes homéotiques. Il savait par conséquent parfaitement que la sénescence de cette mouche était étroitement associée à une diminution de la synthèse protéique, ellemême précédée d'une décroissance de l'activité totale du facteur d'élongation de la traduction EF-1 $\alpha$. Afin de tester l'hypothèse selon laquelle cette décroissance du facteur EF- $1 \alpha$ interviendrait dans la sénescence de la drosophile, les auteurs ont créé des mouches transgéniques exprimant un gène $\mathrm{EF}-1 \alpha$ sous le contrôle du promoteur du gène codant pour hsp70, une protéine du choc thermique. La longévité moyenne des mouches normales et transgéniques à $25^{\circ} \mathrm{C}$ était peu différente, de 38 jours dans le premier cas et de 45 jours dans le second. A $29,5^{\circ} \mathrm{C}$, cependant, la différence entre mouches n'exprimant pas et exprimant le gène $\mathrm{EF}-1 \alpha$ transgénique était plus importante, respectivement de 21 et de 30 jours. La diminution globale de la longévité entre 25 et $29^{\circ} \mathrm{C}$ est un effet général bien connu de la température sur les poïkilothermes et le fait significatif est bien qu'entre 25 et $29,5^{\circ} \mathrm{C}$, alors que le promoteur hsp70 est activé et dirige la synthèse d'une quantité accrue de EF-1 $\alpha$, l'écart s'accroît entre les longévités moyennes des deux types de mouche.

Ainsi apparaît-il qu'avec retard sur d'autres domaines, retard lié à la faiblesse des équipes historiquement engagées dans la recherche des mécanismes moléculaires du vieillissement, la génétique moléculaire va maintenant permettre d'avancer rapidement dans la compréhension des bases génétiques de la longévité et l'identification des gènes impliqués dans la constitution du phénotype sénescent. Puisque nous nous sommes risqué ici à un pronostic sur ce que pourraient être certains de ces processus, c'est avec attention que nous resterons à l'affût de toute donnée nouvelle en ce domaine et que nous en informerons nos lecteurs.

A.K.

1. Sugawara O, Oshimura $M$, Koi $M$, Annab LA, Barrett JC. Induction of cellular senescence in immortalized cells by human chromosome 1. Science $1990 ; 247$ : 707-10.

2. Shepherd JC, Walldorf U, Hug P, Gehring WJ. Fruit flies with additional expression of the elongation factor EF-1 $\alpha$ live longer. Proc Natl Acad Sci USA 1989; 86 : 7520-1.
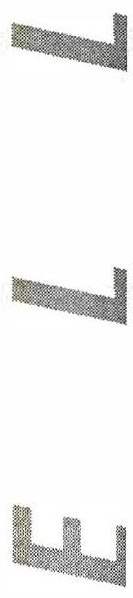